drinks it. The author's final conclusion about $\mathrm{Du}$ Noüy's discussion of time is that he "has gone down clutching a platitude and come up embracing a metaphysical system". In other words, just as physiology suffered for hundreds or even thousands of years from the attempts by Pythagoras to apply the lore of number to it, so the study of senescence has long been bedevilled even to the point of "intellectual disaster" by this type of attempt to describe it in mathematical terms.

But it must not bo thought that Dr. Comfort's book is all motaphysical discussion; far from it--there is (if one may be pardoned such a metaphor at this point) plenty of meat in it. There are sections on the nature and criteria of senescence, maximum longevities among various animals, the maximum life-span in man, the distribution of senescence among vertebrates and invertebrates, senescence in Protozoa, the influence of genetic constitution on senescence and longevity, growth and senescence and the mechanisms of senescence.

Dr. Comfort's book is the most comprehensive review on the subject of senescence that has appeared since 1920. 'The publishers' notice on the cover claims that it has been written to marshal information and suggest lines of investigation for the experimental attacks on old age. In my opinion it fulfils this aim, and it thus becomes an essential part of the bookshelf of anyone interested or working in the field of senescence-or even of 'ageing'. G. H. BOURNE

\section{ENTROPIC ASPECTS OF INFORMATION THEORY}

Science and Information Theory

By Prof. Leon Brillouin. Pp. xvii +320 . (New York: Academic Press, Inc.; London: Academic Books, Ltd., 1956.) 6.80 dollars.

$T^{H E}$ theory of information has not been long in gathering a motley crowd of associates. Too often, in consequence, it has found itself vaguely identified with a wide variety of studies, for which in fact it serves merely as a new and useful tool. "Science and Information Theory", by Prof. L. Brillouin, is a book calculated severely to redress the balance. For Brillouin, information theory means simply what might be called the statistical theory of 'unexpected. ness', developed chiefly by Wiener and Shannon for the purposes of communication engineering.

The mathematical 'unexpectedness' or improbability of a signal, measured logarithmically, (log $1 / p)$, is directly related to the size of the most economical code-symbol to represent it. 'The average unexpectedness $\left(\Sigma p_{i} \log \left(1 / p_{i}\right)\right)$, over a long sequence of signals, indicates the minimum average number of code-symbols necessary for each signal-transmission. Extremely important and illuminating coding theorems follow from this quantification of 'unexpectedness' as the additive measure which has operational significance for the code-designer, and it is not surprising that it is termed the "amount-ofinformation' in the signal.

Unfortunately, as Shannon has been quick to point out, the improbability of a message need have no direct connexion with its logical content--with the number and complexity of the statements it makes. $\mathrm{His}$ measure is not, therefore, as Brillouin (p. 1) would have it, a definition of information, but merely of the unexpectedness of information. As a mathematical index of unexpectedness it can be applied, of course, to other things than messages-to states of thermodynamic systems, for example, in which context the same averago measure has long been known under the name of entropy. The entropy of an under-specified system is numerically proportional to the 'unexpectedness' of tho information which a complete specification would provide.

It is this equivalence of entropy and 'amount of information' (in Shannon's sense) which Prof. Brillouin is chiefly concerned to trace in a wide variety of physical situations, including the mythical case of Maxwell's 'demon'. 'The troatment is lucid, rigorous and illuminating at many points; but the persistent confusion of the concept of information with the unexpectedness of information loads to unnecessary mystification at a number of points. A particularly unconvincing example is the discussion (p. 155) of "free information", as something held "in the mind" and so "not directly connected with any physical system". Metaphysics has its proper place; but this is metaphysics misplaced. 'I'he 'unexpected: ness' which Shannon defined by $\sum p_{i} \log \left(1 / p_{i}\right)$ is not (contra p. 10) an absolute quantity, but a function, defined only in relation to a chosen distribution of prior probabilities or 'ensemble' of events. In physics the distribution may be fixed by the temperature of the system; but in other contexts the probabilities may be determined quite otherwise, and the direct relation of Shannon's measure to physical entropy disappears. Much of the mystery can be removed if the reader will substitute 'unexpectedness' for 'information' wherever the Shannon measure is discussed, and define the relevant ensemble; but one is left with the feeling that many questions would have been differently expressed had the author done the same.

Having equated "Information Theory", with Shannon's theory of unexpectedness, Prof. Brillouin has to disclaim for it any interest in questions of the meaning and value of information. In point of fact, as the proceedings of three IJondon symposia on this subject can illustrate, the general theory of information has a well-defined place for measures of descriptive information-content as well as of unexpectedness. The author does not appear to realize, for example, that the measure developed by Gabor, which he describes on p. 100 , is precisely one of the measures of "structural information-content" (termed "logon: content" by Gabor) for which later (p. 291) he finds no place. "To talk of "inconsistencies" (p. 291) between these complementary measures is simply to reveal misunderstanding of their nature.

As one might expect, the incidental expositions of fundamental physics throughout the book are masterly. It is gratifying, too, to find lucid accounts of work such as Mandelbrot's on word-statistics, and recent developments in the design of error-correcting codes, included among the more conventional topics: Schouten's name, on the other hand, is not men. tioned, despite the similarities between some of his ideas and Brillouin's.

This is a stimulating work-sometimes to insight, occasionally to exasperation. A novice secking to learn the gist and drift of information theory may find easier introductions elsewhere; but for the student who wants to broaden and deepen his thinking in Prof. Brillouin's chosen portions of the subject the book is to be recommended.
D. M. M.CKAY 